\title{
Essential Trace Elements
}

\author{
Contributors: , Patrizia Zavattari ${ }^{1}$, Eleonora Loi ${ }^{2}$, Matteo Serra ${ }^{3}$, (C Paolo Valera ${ }^{4}$ \\ , Davide Firinu ${ }^{5}$, Daniela Cannas ${ }^{6}$ \\ 1, Department of Biomedical Sciences, Unit of Biology and Genetics, University of Cagliari, \\ Cagliari, Italy; pzavattari@unica.it \\ 2, Department of Biomedical Sciences, Unit of Biology and Genetics, University of Cagliari, \\ Cagliari, Italy; eleonora.loi@unica.it \\ 3, Department of Civil, Environmental Engineering and Architecture, University of Cagliari, \\ Cagliari, Italy; srr.mtt.sa@gmail.com \\ 4, Department of Civil, Environmental Engineering and Architecture, University of Cagliari, \\ Cagliari, Italy; pvalera@unica.it \\ 5, davide.firinu@unica.it \\ 6, Department of Biomedical Sciences, Unit of Biology and Genetics, University of Cagliari, \\ Cagliari, Italy; cannasdaniela@libero.it
}

Version received: 20 July 2020 check for updates

Trace elements produce double-edged effects on the lives of animals and particularly of humans. On one hand, these elements represent potentially toxic agents; on the other hand, they are essentially needed to support growth and development and confer protection against disease. The amount taken up and the accumulation in human tissues decisively control whether the exerted effects are toxic or beneficial. This entry provides a brief description of some toxic, likely essential and essential elements and their influence on human health.

\section{Trace Elements}

Trace elements are present in the human body in extremely small quantities of less than $0.01 \%$. They are important for growth, development, maintenance and the recovery of health. They have various roles: some of them are essential components of enzymes, where they attract substrate molecules and facilitate their conversion into final products; others donate or accept electrons in the oxidation-reduction reactions necessary for the production and use of energy in the metabolism; others provide structural stability to some important biological molecules. Finally, some trace elements control important biological processes facilitating the binding of molecules to their receptors on the cell membrane, altering the structure or ionic nature of the membranes to regulate the access of certain molecules into the cell and inducing the expression of genes encoding for proteins involved in various vital processes.

Several studies report evidence of the possible involvement of trace elements in processes leading to an autoimmune response. For example, the causal relationship between mercury and immune diseases is well established and the mechanism has been hypothesized [1].

It has been discovered that mercury, nickel, cadmium, lead, aluminum and arsenic can exert immunotoxic effects through epigenetic mechanisms such as DNA methylation, post-translational modification of histones and miRNAs [2][3]. Furthermore, mercury, a possible risk factor for SLE, has been shown to induce the formation of anti-nuclear antibodies (ANA), hallmark of many autoimmune diseases, in mice ${ }^{[4]}$.

The immunological effects of trace elements include immunomodulation, autoimmunity and allergy. These elements can act as immunosuppressants or as immune adjuvants. Their effects depend on the dose. In fact, their accumulation or deficiency can stimulate an alternative path that could induce the onset of disease. For example, low zinc levels and high levels of copper, manganese and iron participate in the activation of inflammatory responses and responses to oxidative stress induced by the ROS and RNS [5].

Interactions between different oligoelements may play an important role in metabolic disease onset. For example, copper deficiency anemia can develop in people who consume high doses of zinc over a long period of time [6]. Other interactions include the ability of selenium to reduce the toxicity of methylmercury, cadmium ${ }^{[7]}$ and trivalent, 
pentavalent inorganic arsenic through the formation of a conjugated As-Se-glutathyl excreted with bile [8]. In vitro and in vivo studies have shown that small initial doses of cadmium are protective against subsequent high doses of cadmium [9][10]. In fact, cadmium induces the synthesis and storage of low molecular weight proteins (metallothioneins) in the liver and kidneys. Metallothioneins, which are rich in sulfur-containing amino acids, can bind to subsequent doses of mercury or cadmium, thus decreasing their toxicity [9]. High dietary intakes of calcium and magnesium can have a beneficial effect by reducing the gastrointestinal absorption of lead. However, calcium can also reduce the absorption of iron and zinc. Molybdenum can reduce copper retention [11].

The storage of trace elements in non-metabolically active sites or forms is another mechanism that allows the accumulation of elements at dangerous concentrations.

Furthermore, releasing trace elements from a storage site can play an important role in preventing deficiencies. In fact, current evidence suggests that the low affinity copper transporter (CTR2), located in the membranes of intracellular organelles, could serve to release copper from lysosomal deposits or from other vesicles towards intracellular spaces [12]. Similarly, zinc efflux transporters in the Golgi membranes and in the cell membrane play an important role in the homeostatic regulation of the element $[12]$.

The World Health Organization (WHO) ${ }^{[13]}$ classified trace elements into three groups based on their possible nutritional roles:

- potentially toxic elements, e.g., lead $(\mathrm{Pb})$, cadmium $(\mathrm{Cd})$, fluorine $(\mathrm{F})$, mercury $(\mathrm{Hg})$, arsenic (As), aluminum (Al), barium $(\mathrm{Ba})$, lithium $(\mathrm{Li})$, tin $(\mathrm{Sn})$;

- elements of probable physiological importance, e.g., manganese (Mn), silicon (Si), nickel (Ni), boron (B), vanadium $(\mathrm{V})$;

- essential elements, e.g., chromium ( $\mathrm{Cr}$ ), copper (Cu), zinc (Zn), selenium (Se), molybdenum (Mb), cobalt (Co), iodine (I).

The elements mentioned are introduced mainly with food.

\subsection{Potentially Toxic Elements}

\subsubsection{Lead}

Lead $(\mathrm{Pb})$ is one of the most important toxic elements. It has been used for decades for many technological processes, including the production of paints, gasoline and aviation fuel. The previous use of carbonate and oxide lead in these products represents the main source of exposure to this metal that is not degradable and remains in the environment as dust, in the soil and paint in old houses.

Lead toxicity is based on molecular mimicry with cellular cations and the formation of ROS. It replaces zinc and calcium in proteins involved in different biological processes, consequently altering protein structure and function [14].

Lead passes through the placenta, determining an increase in the blood levels of the fetus nearly identical to that in the maternal blood ${ }^{[15}$. Lead toxicity affects almost every organ in the body, but the central nervous system is particularly sensitive to lead effects in both children and adults. Learning deficits and behavioral problems are serious effects of lead poisoning in children, while in adults, neuropathies, chronic nephropathies, anemia, hypertension and toxicity related to the reproductive organs are of particular importance [16]. Another target of lead toxicity is the immune system. According to Fenga et al. (2017), lead enhances Th2 cell development, affecting Th1 cell proliferation and leading to high levels of IgE and inflammatory cytokines [17]. However, other authors have demonstrated that there are not changes in cytokine levels related to the Th1-, Th2- and Th17-mediated immune responses after short-term exposure to lead, in contrast to chronic exposure [18]. Therefore, lead alters Th cell functions, increasing the susceptibility to autoimmune diseases and hypersensitivity. For example, one study found a positive correlation between exposure to absorbable lead in soil and MS prevalence in Iran [19].

\subsubsection{Cadmium}




\section{Encyclopedia}

Cadmium $(\mathrm{Cd})$ is a toxic transition metal. Its industrial use was irrelevant up until 50 years ago. About $75 \%$ of the cadmium produced is used in batteries. It is also used as a pigment in paints and as a stabilizer in plastics. A potential source of cadmium is represented by the extraction of zinc and lead in mines, where lead is a secondary product. The natural presence of cadmium in zinc and lead deposits is well known.

The primary source of cadmium exposure is food, particularly cereals, leafy fruits and vegetables, crustaceans and liver and kidneys of animals, as well as contaminated drinks and cigarette smoke. Cadmium transplacental passage is not easy; however, since the absorption of micronutrients increases in pregnancy, cadmium absorption increases and accumulates at high concentrations. Cadmium exposure has been associated with nephrotoxicity, hepatotoxicity and effects on the immune system, bones and male reproductive physiology.

Several studies have shown that cadmium is an immunomodulator. It stimulates the production of Th2 cells. Stimulated macrophages and monocytes respond with the release of ROS and TNF $\alpha$ and production of the inducible nitric oxide (NO) enzyme synthase. Nitrogen oxides are known to regulate the proliferative response of lymphocytes [7]. Cadmium has been shown to inhibit glutathione reductase enzymes, implicated in the defense against free radicals, and the enzyme thioredoxin reductase, an oxidoreductase that reduces protein thiols and has an important role in regulating the redox state of cells $[20]$. Furthermore, an in vitro study has shown that low doses of cadmium stimulate the immune system, while higher concentrations inhibit immune responses [21]. This could be in agreement with the results of an Iranian study in which a high concentration of absorbable cadmium in the soil was associated with a lower prevalence of MS ${ }^{[19]}$.

\subsubsection{Barium}

Barium (Ba) constitutes about $0.05 \%$ of the Earth's crust. Exposure to this element deserves particular attention because it is commonly found in surface waters and can be released into the environment by the natural breakdown of rocks and minerals or as polluting waste from industry and human activities.

The toxicity of barium-containing compounds depends on solubility, i.e., soluble salts are more toxic than insoluble salts because they can be absorbed through the skin or inhaled. A US study reported the presence of $10 \mathrm{ppm}$ (parts per million) of barium in wheat and corn crops; milk, potatoes and flour were the main sources of barium in the American diet [22].

The mechanism of action of $\mathrm{Ba}$ involves blocking $\mathrm{K}^{+}$efflux channels in the cell membrane, with a consequent increase in intracellular $\mathrm{K}+$ levels and extracellular hypokalemia $[23]$.

It can have an effect on skeletal muscles, smooth muscle and myocardial excitability and can lead to secondary respiratory paralysis and heart disease.

Although barium is found in low concentrations in the environment, the health consequences of chronic exposure have yet to be analyzed. A correlation study between geochemical data in Europe and the incidence of MS and T1D found a positive correlation between barium and sodium oxide present in soil and river sediments and these autoimmune diseases [24].

\subsubsection{Lithium}

Lithium ( $\mathrm{Li}$ ) is a moderately abundant alkaline metal present in the Earth's crust in an amount equal to $20 \mathrm{ppm}$. Lithium is easily absorbed by plants and its quantity varies widely, reaching $30 \mathrm{ppm}$ in some cases. It is used for the production of alkaline batteries, soaps and large refrigeration and air conditioning systems. Lithium carbonate is used as an effective drug for the treatment of bipolar disorder.

Lithium can be absorbed by the inhalation of its aerosol and by ingestion. It was shown that treatment with lithium reduced MS symptoms in MS mouse models [25].

\subsubsection{Mercury}

Mercury $(\mathrm{Hg})$ is found in rocks in the Earth's crust and occurs in coal and other fossil fuels. It is a common 
environmental pollutant. The most common sources of exposure are the consumption of fish and shellfish contaminated with methylmercury and the inhalation of mercury vapors in industrial environments—for example, during the preparation of dental amalgam [26].

The immunotoxic effects of mercury have been observed in humans and animal models. It has been shown that subtoxic doses of mercury may induce a systemic autoimmune syndrome in mouse models [27]. Low doses of mercury and methylmercury cause immunosuppression, reducing Th1 responses and increasing those of Th2 [28][29].

It has been suggested that mercury may have a role in accelerating or aggravating pre-existing systemic autoimmune conditions $[30]$.

\subsection{Likely Essential Elements}

\section{Silicon}

Silicon ( $\mathrm{Si}$ ) is a very abundant element in the Earth's crust, second only to oxygen, making up about $28 \%$ of the Earth's weight. It is not found in nature in its free state but in the form of oxides and silicates. Small quantities (1-10 ppm) of silica and silicates dissolved or in colloidal suspensions are present in surface waters. It is possible to find silicon at different concentrations in various species of plants; for example, it is detectable at concentrations close to $1.2 \%$ in Zea mays.

In vivo studies have shown lipid peroxidation, oxidative stress, an increase in $\operatorname{lgM}$, serum IgG and the presence of ANA in mice exposed to silica, supporting its role as an adjuvant of T lymphocytes and as a possible factor triggering autoimmune diseases such as SLE and glomerulonephritis [31].

An association between systemic immune diseases and occupational exposure to silica dust has been observed 32 . Silica-derived polymers such as silicone elastomer have been increasingly recognized as potential inducers of autoimmune and autoinflammatory (linked to innate immunity stimulation) syndromes [33][34].

\subsection{Essential Elements}

\subsubsection{Zinc}

Zinc $(\mathrm{Zn})$ is an essential trace element, ubiquitous in the environment and widely distributed in the body.

The dietary requirement for zinc is between 6.2 and $10.2 \mathrm{mg} /$ day for women, between 7.5 and $12.7 \mathrm{mg} / \mathrm{day}$ for men and between 2.4 and $11.8 \mathrm{mg} /$ day for children [35]. Rich sources of $\mathrm{Zn}$ in the diet are meat, milk, legumes, eggs, fish and cereals. Phytates (present in legumes, nuts and seeds), calcium and phosphates reduce zinc absorption. On the other hand, amino acids, picolinic acid and prostaglandin E2 can increase its absorption. Zinc needs increase up to two times during pregnancy and breastfeeding. In fact, zinc is lost in quantities of up to 2 mg per day until to 2 months after childbirth. Additionally, preterm infants require higher zinc levels due to inadequate deposits, reduced intestinal absorption and increased metabolic rate.

It has been observed that the fraction of zinc absorbed progressively decreases as zinc intake increases. This is due to the fact that $\mathrm{Zn}$ is an effective inducer of metallothionein synthesis, and when metallothionein in the intestinal cells is saturated, the absorption of $\mathrm{Zn}$ is reduced [35].

Zinc is predominantly found in muscles, bones, skin/hair, the liver and the pancreas. About $99 \%$ of zinc is intracellular and is distributed in the cytoplasm (50\%), in the nucleus (30-40\%) and in the cell membrane (10\%), while the rest (about $1 \mathrm{mg} / \mathrm{L})$ is bound to albumin in plasma (60-80\%) [5] .

Zinc is involved in a wide range of vital catalytic, structural and regulatory physiological processes and is required by more than 300 enzymes for their catalytic activation. It is involved in DNA, RNA and protein syntheses and is the cofactor of several transcription factors modulating the expression of zinc sensitive genes [ $\underline{36}$. Furthermore, Zn binds to over 2500 proteins, maintaining their structural integrity and regulating their functions. It is one of the cofactors of the enzyme Cu/Zn superoxide dismutase (SOD), which plays a fundamental role in the removal of ROS and reduction of 


\section{Encyclopedia}

lipid peroxidation.

Zinc also plays an immune, anti-inflammatory and antioxidant role. It mediates innate immunity and influences acquired immunity by activating $T$ lymphocytes and regulating the production of Th1 cytokines, B lymphocytes and antibodies. It is also used by macrophages for phagocytosis and cytokine production [36].

A recent meta-analysis has shown that low levels of zinchemia (in serum and plasma) are often observed in patients with autoimmune diseases [37. Low zinc levels are known to contribute to immune defects associated with malnutrition [38]. In particular, a systemic zinc deficiency is associated with inflammation states, producing effects on the immune system. Interestingly, experiments conducted on cells, synoviocytes isolated from rheumatoid arthritis patients, have shown that the production of inflammation mediated cytokines IL-17/TNF in turn stimulates zinc uptake by the synoviocytes, thus increasing even more the inflammation, in a feedback loop between inflammation and zinc uptake [39].

As previously reported, $\mathrm{Zn}$ reduces the generation of ROS involved in the activation of NF-kB, a modulator of the immune response to infections whose dysfunction can cause autoimmune diseases and tumors. NF-kB inhibition results in the reduced generation of inflammatory cytokines and adhesion molecules [5].

Zinc deficiency induces thymic atrophy and reduces the activity of serum thymulin, a thymic zinc-dependent hormone necessary for the maturation and differentiation of $T$ helper lymphocytes. The result is a decrease in Th1 cytokines, with a shift in activity towards Th2 lymphocytes and a reduction in the activity of natural-killer (NK) and cytotoxic T cells. Therefore, $\mathrm{Zn}$ deficiency induces an imbalance between Th1 and Th2 cell functions and between Treg lymphocytes and pro-inflammatory T cells, as well as the induction of Th17 lymphocyte activity, the main mechanisms that contribute to autoimmune disease pathogenesis. [5]. The proliferation of pre-activated human $\mathrm{T}$ cells and Th1/Th2/Th17 cytokine production may be suppressed by zinc aspartate [40].

A study has shown that the addition of $\mathrm{Zn}$, in combination with probiotic complex and coenzyme Q1, to the diet of an animal model of arthritis suppressed the differentiation of Th17 lymphocytes [41]. Another study demonstrated that $\mathrm{Zn}$ suppressed Th17 development by inhibiting STAT3 activation in a mouse model of rheumatoid arthritis [42]. Zinc also induces a variety of other proinflammatory responses in T cells and B cells [43][44][45].

Studies in Sardinia and Sweden have shown that low concentrations of $\mathrm{Zn}$ in soils and drinking water were associated with a higher risk of T1D, suggesting that this metal has a protective role ${ }^{446]}$.

\subsubsection{Copper}

Copper $(\mathrm{Cu})$ is the third most abundant essential trace element in the human body, after iron and zinc, constituting 75-100 mg of the total quantity. The dietary requirements for copper are $1.6 \mathrm{mg} /$ day for men, $1.3 \mathrm{mg} /$ day for women and $1 \mathrm{mg} /$ day for children [47].

$\mathrm{Cu}$ is widely distributed in nature and is found at a concentration of about $55 \mathrm{ppm}$ in the Earth's crust (77). It is almost always found in the form of minerals, such as sulphides, oxides, carbonates, silicates or, more rarely, in their native states. The most abundant minerals are copper and iron sulphides (chalcopyrite).

Food, drinks and water are the main source of exposure of the population to copper sulphate. Copper is contained in greater quantities in meat, liver and kidneys, in cereals, mollusks and in some fruits (avocado, walnuts, hazelnuts and dried grapes).

Copper exists in two oxidation states, as $\mathrm{Cu}$ (I) or $\mathrm{Cu}$ (II), and this ability to gain or lose an electron is at the basis of its role in the energy transfer processes in biological systems and in cellular respiration.

Many enzymes require copper as a cofactor, particularly those involved in iron metabolism, in the synthesis of neurotransmitters, in energy metabolism and in the cross-linking of collagen and elastin.

Copper deficiency symptoms have been observed in a X-linked recessive disease due to mutations of the copper 


\section{Encyclopedia}

transporter ATP7A. They include anemia, hypercholesterolaemia, metabolic syndrome, reduced glucose tolerance, hypopigmentation of the skin and hair, leukopenia, neutropenia, myelodysplasia and, in most patients, neurological effects, most commonly due to neuromyelopathy [48][49]. It has been hypothesized that anemia associated with copper deficiency is due to defective iron mobilization, resulting from reduced ceruloplasmin activity [49]. This enzyme, with its ferroxidase action, is fundamental for the transformation of $\mathrm{Fe}^{2+}$ to $\mathrm{Fe}^{3+}$, an indispensable step for the incorporation of iron into the circulating transferrin to avoid the toxicity of the free metal involved in the production of free radicals. Furthermore, copper deficiency has been associated with changes in immune and bone function. In particular, a reduced number of leukocytes and neutrophils and reduced antioxidant activity of Cu/Zn SOD, as well as the presence of lipid peroxidation, have been detected [48].

Since copper is involved in the synthesis of the myelin sheath, its deficiency can potentially cause myelopathy $[50]$. Moreover, copper has a regulatory role in cell growth and in maintaining homeostasis in the immune system. In particular copper sulphate seems to exert beneficial effects in T1D mouse models, both by directly reducing the amount of free radicals and by lowering blood glucose levels [51].

\subsubsection{Chromium}

Chromium ( $\mathrm{Cr}$ ) is an essential nutrient involved in protein, lipid and carbohydrate metabolism. The dietary requirement for chromium is 20-35 $\mathrm{gg} /$ day [52]. Chromium is naturally present in trivalent, $\mathrm{Cr}$ (III), and hexavalent, $\mathrm{Cr}(\mathrm{VI})$, forms. Cr (VI) has been extensively used in the paint, steel manufacturing and leather industries. The association between $\mathrm{Cr}$ (VI) toxicity and lung cancer in stainless workers is well established [53]. Moreover, it has been demonstrated that $\mathrm{Cr}$ (VI) induces oxidative stress by increasing the production of ROS [54].

On the other hand, $\mathrm{Cr}$ (III) salts have been shown to possess beneficial effects as nutritional supplements in animals and humans [55].

Chromium deficiency has been observed in diabetic patients receiving chronic total parenteral nutrition. Chromium supplementation resulted in improved glucose tolerance [56].

\section{References}

1. Schiraldi, M.; Monestier, M. How can a chemical element elicit complex immunopathology? Lessons from mercury-induced autoimmunity. Trends Immunol. 2009, 30, 502-509.

2. Martinez-Zamudio, R.; Ha, H.C. Environmental epigenetics in metal exposure. Epigenetics 2011, 6, 820-827.

3. Hou, L.; Zhang, X.; Wang, D.; Baccarelli, A. Environmental chemical exposures and human epigenetics. Int. J. Epidemiol. 2012, 41, 79-105.

4. Abedi-Valugerdi, M.; Hu, H.; Gö, G.; Mö, M. Mercury-induced anti-nucleolar autoantibodies can transgress the membrane of living cells in vivo and in vitro. Int. Immunol. 1999, 11, 605-615.

5. Mezzaroba, L.; Alfieri, D.F.; Colado Simão, A.N.; Vissoci Reiche, E.M. The role of zinc, copper, manganese and iron in neurodegenerative diseases. Neurotoxicology 2019, 74, 230-241.

6. Hoffman, H.N.; Phyliky, R.L.; Fleming, C.R. Zinc-induced copper deficiency. Gastroenterology 1988, 94, 508-512.

7. Marth, E.; Barth, S.; Jelovcan, S. Influence of cadmium on the immune system. Description of stimulating reactions. Cent. Eur. J. Public Health 2000, 8, 40-44

8. Zeng, H.; Uthus, E.O.; Combs, G.F. Mechanistic aspects of the interaction between selenium and arsenic. J. Inorg. Biochem. 2005, 99, 1269-1274.

9. Gaddipati, J.P.; Rajeshkumar, N.V.; Grove, J.C.; Maharaj, S.V.M.; Centeno, J.A.; Maheshwari, R.K.; Jonas, W.B. Low-Dose Cadmium Exposure Reduces Human Prostate Cell Transformation in Culture and Up-Regulates Metallothionein and MT-1G mRNA. Nonlinearity Biol. Toxicol. Med. 2003, 1, 154014203914343.

10. Herkovits, J.; Perez-Coll, C.S. Increased resistance against cadmium toxicity by means of pretreatment with low cadmium/zinc concentrations in Bufo arenarum embryos. Biol. Trace Elem. Res. 1995, 49, 171-175.

11. Bogden, J.D. The Essential Trace Elements and Minerals. In Clinical Nutrition of the Essential Trace Elements and Minerals; Humana Press: Totowa, US, 2000; pp. 3-9.

12. Martinez-Finley, E.J.; Chakraborty, S.; Fretham, S.J.B.; Aschner, M. Cellular transport and homeostasis of essential and nonessential metals. Metallomics 2012, 4, 593-605.

13. Squitti, R.; Negrouk, V.; Perera, M.; Llabre, M.M.; Ricordi, C.; Rongioletti, M.C.A.; Mendez, A.J. Serum copper profile in patients with type 1 diabetes in comparison to other metals. J. Trace Elem. Med. Biol. 2019, 56, 156-161.

14. Garza, A.; Vega, R.; Soto, E. Cellular mechanisms of lead neurotoxicity. Med. Sci. Monit. 2006, 12, RA57-RA65. 
15. Goyer, R.A. Transplacental transport of lead. Environ. Health Perspect. 1990, 89, 101-105.

16. Patrick, L. Lead toxicity, a review of the literature. Part I: Exposure, evaluation, and treatment. Altern. Med. Rev. 2006, 11, 2-22.

17. Fenga, C.; Gangemi, S.; Di Salvatore, V.; Falzone, L.; Libra, M. Immunological effects of occupational exposure to lead (Review). Mol. Med. Rep. 2017, 15, 3355-3360.

18. Dobrakowski, M.; Boroń, M.; Czuba, Z.P.; Kasperczyk, A.; Machoń-Grecka, A.; Kasperczyk, S. Cytokines related to three major types of cell-mediated immunity in short- and long-term exposures to lead compounds. J. Immunotoxicol. 2016, 13, 770-774.

19. Etemadifar, M.; Mehrabi, B.; Kiani-Peykani, R.; Abtahi, S.H.; Nekouie-Isfahani, K.; Ramagopalan, S.V.; Fereidan-Esfahani, M. Soil heavy metals are associated with the distribution of multiple sclerosis in Isfahan, Iran. Acta Neurol. Scand. 2016, 134, 292-299.

20. Azeh Engwa, G.; Udoka Ferdinand, P.; Nweke Nwalo, F.; Unachukwu, M.N. Mechanism and Health Effects of Heavy Metal Toxicity in Humans. In Poisoning in the Modern World-New Tricks for an Old Dog? IntechOpen: London, UK, 2019.

21. Krocova, Z.; MacEla, A.; Kroca, M.; Hernychova, L. The immunomodulatory effect(s) of lead and cadmium on the cells of immune system in vitro. Toxicol. Vitr. 2000, 14, 33-40.

22. Calabrese, E.J.; Canada, A.T.; Sacco, C. Trace Elements and Public Health. Annu. Rev. Public Health 1985, 6, $131-146$.

23. McNeill, I.R.; Isoardi, K.Z. Barium poisoning: An uncommon cause of severe hypokalemia. Toxicol. Commun. 2019 , 3, 88-90.

24. Valera, P.; Zavattari, P.; Albanese, S.; Cicchella, D.; Dinelli, E.; Lima, A.; De Vivo, B. A correlation study between multiple sclerosis and type 1 diabetes incidences and geochemical data in Europe. Environ. Geochem. Health 2014, 36, 79-98.

25. De Sarno, P.; Axtell, R.C.; Raman, C.; Roth, K.A.; Alessi, D.R.; Jope, R.S. Lithium Prevents and Ameliorates Experimental Autoimmune Encephalomyelitis. J. Immunol. 2008, 181, 338-345.

26. Mehri, A. Trace elements in human nutrition (II)—An update. Int. J. Prev. Med. 2020, 11, 2.

27. WHO. Environmental Health Criteria 236 PRINCIPLES AND METHODS FOR ASSESSING AUTOIMMUNITY ASSOCIATED WITH EXPOSURE TO First Draft Prepared by the World Health Organization Collaborating; World Health Organization: Geneva, Switzerland, 2006; Volume 4, pp. 1-4.

28. Bagenstose, L.M.; Mentink-Kane, M.M.; Brittingham, A.; Mosser, D.M.; Monestier, M. Mercury enhances susceptibility to murine leishmaniasis. Parasite Immunol. 2001, 23, 633-640.

29. Silbergeld, E.K.; Devine, P.J. Mercury-Are we studying the right endpoints and mechanisms. Fuel Process. Technol. 2000, 65, 3542.

30. Havarinasab, S.; Lambertsson, L.; Qvarnström, J.; Hultman, P. Dose-response study of thimerosal-induced murine systemic autoimmunity. Toxicol. Appl. Pharmacol. 2004, 194, 169-179.

31. Mayeux, J.M.; Escalante, G.M.; Christy, J.M.; Pawar, R.D.; Kono, D.H.; Pollard, K.M. Silicosis and Silica-Induced Autoimmunity in the Diversity Outbred Mouse. Front. Immunol. 2018, 9, 874.

32. Miller, F.W.; Alfredsson, L.; Costenbader, K.H.; Kamen, D.L.; Nelson, L.M.; Norris, J.M.; De Roos, A.J. Epidemiology of environmental exposures and human autoimmune diseases: Findings from a National Institute of Environmental Health Sciences Expert Panel Workshop. J. Autoimmun. 2012, 39, 259-271.

33. Zandman-Goddard, G.; Blank, M.; Ehrenfeld, M.; Gilburd, B.; Peter, J.; Shoenfeld, Y. A comparison of autoantibody production in asymptomatic and symptomatic women with silicone breast implants. J. Rheumatol. 1999, 26, 73-77.

34. Soriano, A.; Butnaru, D.; Shoenfeld, Y. Long-term inflammatory conditions following silicone exposure: The expanding spectrum of the autoimmune/inflammatory syndrome induced by adjuvants (ASIA). Clin. Exp. Rheumatol. 2014, 32, 151-154.

35. European Food Safety Authority. Scientific Opinion on Dietary Reference Values for zinc. EFSA J. 2014, $12,3844$.

36. Tuerk, M.J.; Fazel, N. Zinc deficiency. Curr. Opin. Gastroenterol. 2009, 25, 136-143.

37. Sanna, A.; Firinu, D.; Zavattari, P.; Valera, P. Zinc status and autoimmunity: A systematic review and meta-analysis. Nutrients 2018 , $10,68$.

38. Plum, L.M.; Rink, L.; Hajo, H. The essential toxin: Impact of zinc on human health. Int. J. Environ. Res. Public Health 2010, 7, 13421365.

39. Bonaventura, P.; Lamboux, A.; Albarède, F.; Miossec, P. A Feedback Loop between Inflammation and Zn Uptake. PLoS ONE 2016, $11, \mathrm{e} 0147146$.

40. Guttek, K.; Wagenbrett, L.; Reinhold, A.; Grüngreiff, K.; Reinhold, D. Zinc aspartate suppresses proliferation and Th1/Th2/Th17 cytokine production of pre-activated human T cells in vitro. J. Trace Elem. Med. Biol. 2018, 49, 86-90.

41. Lee, S.Y.; Lee, S.H.; Jhun, J.; Seo, H.B.; Jung, K.A.; Yang, C.W.; Park, S.H.; Cho, M. La A Combination with Probiotic Complex, Zinc, and Coenzyme Q10 Attenuates Autoimmune Arthritis by Regulation of Th17/Treg Balance. J. Med. Food 2018, 21, 39-46.

42. Kitabayashi, C.; Fukada, T.; Kanamoto, M.; Ohashi, W.; Hojyo, S.; Atsumi, T.; Ueda, N.; Azuma, I.; Hirota, H.; Murakami, M.; et al. Zinc suppresses Th 17 development via inhibition of STAT3 activation. Int. Immunol. 2010, 22, 375-386.

43. Lee, H.; Kim, B.; Choi, Y.H.; Hwang, Y.; Kim, D.H.; Cho, S.; Hong, S.J.; Lee, W.W. Inhibition of interleukin-1 $\beta$-mediated interleukin-1 receptor-associated kinase 4 phosphorylation by zinc leads to repression of memory T helper type 17 response in humans. Immunology 2015, 146, 645-656.

44. Hojyo, S.; Miyai, T.; Fujishiro, H.; Kawamura, M.; Yasuda, T.; Hijikata, A.; Bin, B.H.; Irié, T.; Tanaka, J.; Atsumi, T.; et al. Zinc transporter SLC39A10/ZIP10 controls humoral immunity by modulating B-cell receptor signal strength. Proc. Natl. Acad. Sci. USA $2014,111,11786-11791$. 
45. Schubert, C.; Guttek, K.; Grüngreiff, K.; Thielitz, A.; Bühling, F.; Reinhold, A.; Brocke, S.; Reinhold, D. Oral zinc aspartate treats experimental autoimmune encephalomyelitis. BioMetals 2014, 27, 1249-1262.

46. Valera, P.; Zavattari, P.; Sanna, A.; Pretti, S.; Marcello, A.; Mannu, C.; Targhetta, C.; Bruno, G.; Songini, M. Zinc and Other Metals Deficiencies and Risk of Type 1 Diabetes: An Ecological Study in the High Risk Sardinia Island. PLoS ONE 2015, 10, e0141262.

47. European Food Safety Authority. Scientific Opinion on Dietary Reference Values for copper. EFSA J. 2015, $13,4253$.

48. Uriu-Adams, J.Y.; Keen, C.L. Copper, oxidative stress, and human health. Mol. Aspects Med. 2005, 26, $268-298$.

49. Olivares, M.; Uauy, R. Copper as an essential nutrient. Am. J. Clin. Nutr. 1996, 63, 7918-7924.

50. Sedighi, B.; Ebrahimi, H.A.; Haghdoost, A.A.; Abotorabi, M. Comparison of serum levels of copper and zinc among multiple sclerosis patients and control group. Iran. J. Neurol. 2013, 12, 125.

51. Sitasawad, S.; Deshpande, M.; Katdare, M.; Tirth, S.; Parab, P. Beneficial effect of supplementation with copper sulfate on STZdiabetic mice (IDDM). Diabetes Res. Clin. Pract. 2001, 52, 77-84.

52. Food and Nutrition Board, Institute of Medicine. Dietary Reference Intakes for Vitamin A, Vitamin K, Arsenic, Boron, Chromium, Copper, lodine, Iron, Manganese, Molybdenum, Nickel, Silicon, Vanadium, and Zinc; National Academies Press: Washington, DC, USA, 2001

53. Gad, S.C. Acute and chronic systemic chromium toxicity. Sci. Total Environ. 1989, 86, 149-157.

54. Shrivastava, R.; Upreti, R..; Seth, P..; Chaturvedi, U. Effects of chromium on the immune system. FEMS Immunol. Med. Microbiol. 2002, 34, 1-7.

55. Anderson, R.A. Chromium in the prevention and control of diabetes. Diabetes Metab. 2000, 26, 22-27.

56. Brown, R.O.; Forloines-Lynn, S.; Cross, R.E.; Heizer, W.D. Chromium deficiency after long-term total parenteral nutrition. Dig. Dis. Sci. 1986, 31, 661-664.

\section{Keywords}

Trace Elements; Potentially Toxic Elements; Likely Essential Elements; Essential Elements; Lead; Cadmium; Barium; Mercury; Zinc; Copper

\section{DOI Information}

DOI: 10.32545/encyclopedia202007.0015.v3 | ه PDF [ 704kb, updated 23 July 2020 ]

(C) 2020 by the author(s). Distribute under a Creative Commans CC BY license 